\title{
Gelatin-methacrylamide gel loaded with microspheres to deliver GDNF in bilayer collagen conduit promoting sciatic nerve growth
}

This article was published in the following Dove Press journal:

International Journal of Nanomedicine

I April 2016

Number of times this article has been viewed

\author{
Hai Zhuang ${ }^{1-3}$ \\ Shoushan Bu' \\ Lei Hua' \\ Mohammad A Darabi ${ }^{2,3}$ \\ Xiaojian $\mathrm{Cao}^{4}$ \\ Malcolm Xing 2,3 \\ 'Department of Stomatology, The \\ First Affiliated Hospital with Nanjing \\ Medical University, Nanjing, Jiangsu \\ Province, People's Republic of \\ China; ${ }^{2}$ Department of Mechanical \\ Engineering, Biochemistry \& Medical \\ Genetics, ${ }^{3}$ Children's Hospital \\ Research Institute of Manitoba, \\ University of Manitoba, Winnipeg, MB, \\ Canada; ${ }^{4}$ Department of Orthopedics, \\ The First Affiliated Hospital with \\ Nanjing Medical University, Nanjing, \\ Jiangsu Province, People's Republic \\ of China
}

\begin{abstract}
In this study, we fabricated glial cell-line derived neurotrophic factor (GDNF)-loaded microspheres, then seeded the microspheres in gelatin-methacrylamide hydrogel, which was finally integrated with the commercial bilayer collagen membrane (Bio-Gide ${ }^{\circledR}$ ). The novel composite of nerve conduit was employed to bridge a $10 \mathrm{~mm}$ long sciatic nerve defect in a rat. GDNF-loaded gelatin microspheres had a smooth surface with an average diameter of $3.9 \pm 1.8 \mu \mathrm{m}$. Scanning electron microscopy showed that microspheres were uniformly distributed in both the GelMA gel and the layered structure. Using enzyme-linked immunosorbent assay, in vitro release studies ( $\mathrm{pH} 7.4$ ) of GDNF from microspheres exhibited an initial burst release during the first 3 days $(18.0 \% \pm 1.3 \%)$, and then, a prolonged-release profile extended to 32 days. However, in an acidic condition ( $\mathrm{pH} 2.5$ ), the initial release percentage of GDNF was up to $91.2 \% \pm 0.9 \%$ within 4 hours and the cumulative release percentage of GDNF was $99.2 \% \pm 0.2 \%$ at 48 hours. Then the composite conduct was implanted in a $10 \mathrm{~mm}$ critical defect gap of sciatic nerve in a rat. We found that the nerve was regenerated in both conduit and autograft (AG) groups. A combination of electrophysiological assessment and histomorphometry analysis of regenerated nerves showed that axonal regeneration and functional recovery in collagen tube filled with GDNF-loaded microspheres $(\mathrm{GM}+\mathrm{CT})$ group were similar to AG group $(P>0.05)$. Most myelinated nerves were matured and arranged densely with a uniform structure of myelin in a neat pattern along the long axis in the AG and GM + CT groups, however, regenerated nerve was absent in the BLANK group, left the $10 \mathrm{~mm}$ gap empty after resection, and the nerve fiber exhibited a disordered arrangement in the collagen tube group. These results indicated that the hybrid system of bilayer collagen conduit and GDNF-loaded gelatin microspheres combined with gelatin-methacrylamide hydrogels could serve as a new biodegradable artificial nerve guide for nerve tissue engineering.
\end{abstract}

Keywords: controlled release of GDNF, gelatin microspheres, biodegradable nerve conduit, gelatin-methacrylamide hydrogel, sciatic nerve regeneration

\section{Introduction}

Injuries of peripheral nervous system (PNS) lead to permanent functional loss of target tissue and neuropathic pain and decrease life quality. The regeneration of defected or damaged peripheral nerve has become a difficult and challenging problem. So far, existing problems in repairing PNS include (1) difficulty in building appropriate microenvironment for nerve regeneration, such as lack of required growth factors, and (2) diminishing neuronal apoptosis and muscle atrophy. ${ }^{1}$ For peripheral nerve repair, nerve autograft has been considered as the "gold standard" for the restoration of nerve regeneration. ${ }^{2}$ But autograft is limited by the short age availability of donor nerves, wound pain, increased morbidity, and scar formation at the donor site. ${ }^{3}$ 
To seek alternatives for autograft, biodegradable artificial nerve guide scaffolds (NGSs) have attracted considerable interest. NGS, as a physical scaffold, generally can be loaded with cells and growth factors for nerve regeneration. It is facile to modify its conduit sizes and chemical and physical properties by using different materials and preparation methods. Many studies show that NGSs are used hopefully to create a favorable microenvironment to regenerate axons and to support the cellular growth and migration. ${ }^{4,5}$

Material selection and optimization of NGS is important for an ultimately good restoration outcome. NGS should meet the following requirements: bridging the gap of defected nerve, protecting the regenerating axons from invasion by the surrounding tissue, guiding the regenerating axons into the distal nerve stump, and scaffold degrading with continuous nerve regeneration. ${ }^{1,2,6}$ Therefore, an ideal material of NGS should be biodegradable and biocompatible, and should have proper mechanical strength and no immunogenic response. ${ }^{2}$ Varieties of biopolymers have been employed for nerve conduit as an alternative to autografts. Collagen, a primary constituent of natural extracellular matrix, has been employed as material of scaffolds for tissue engineering due to many excellent properties: low antigenicity, promotion of cell attachment, and full bioabsorption. ${ }^{7,8}$ Bio-Gide $^{\circledR}$ is a kind of bilayer collagen membrane derived from porcine dermis. ${ }^{9}$ Moreover, it has been approved by the US Food and Drug Administration and widely used in clinics for guided tissue/bone regeneration. Bilayer structure not only prevents ingrowth of soft tissue into the lumen due to outer layer with small porosity but also promotes cell attachment attributed to inner layer with large aperture. ${ }^{9,10}$ Many studies indicate that the scaffold can promote nerve regeneration individually. ${ }^{11-13}$ However, if combined with growth factors or seed cells, nerve regeneration will get better outcome. ${ }^{8,14}$ It is an important yet-to-be-solved problem about how to combine scaffold with growth factors or seed cells to establish an optimal microenvironment efficiently and effectively.

Gelatin, which is essentially denatured collagen, can tune its degradation rate via crosslinking and functionalized side groups of polymer chains. It has become a favorite scaffolding material in tissue engineering. ${ }^{15-20}$ For instance, gelatin can form thermos-reversible hydrogels below their upper critical solution temperature of $25^{\circ} \mathrm{C}-35^{\circ} \mathrm{C} .{ }^{21}$ Gelatinmethacrylamide (GelMA), modification of gelatin with unsaturated methacrylate groups, can be copolymerized to form hydrogel via light or chemical initiators under mild conditions with low cytotoxicity. ${ }^{21-23}$

Growth factors play a key role in the regeneration of damaged tissues by stimulating cellular activities such as cell migration, proliferation, and differentiation. However, growth factors, released directly without protection, will be quickly degraded and deactivated by enzymes and other physicalchemical effectors in vivo and in vitro. So polymeric local delivery systems are used for controlled release of growth factors. ${ }^{19,24}$ Theoretically, growth factors and polymers can form a relatively stable controlled-release system, under an appropriate environmental condition, for instance, via the interaction of static electricity between polymers and growth factors. Glial cell-line derived neurotrophic factor (GDNF) is a type of neurotrophic factor secreted by Schwann cells (SCs) after nerve injury. GDNF can improve motor and sensory nerve survival, influence SCs migration, improve the survival of dopamine neurons, and promote peripheral nerve regeneration..$^{25,26}$

To this end, we fabricated GDNF-loaded gelatin microspheres, and the microspheres were formed via complexation procedures between positively charged GDNF (isoelectric point $[\mathrm{pI}]=9.23$ ) and negatively charged Type B gelatin $(\mathrm{pI}=4.7-5.2)$ in a neutral condition. We then studied the releasing property of GDNF-loaded gelatin microspheres in vitro via enzyme-linked immunosorbent assay. We immersed the microspheres into GelMA hydrogel, which was then coated onto nerve conduit for bridging a $10 \mathrm{~mm}$ long sciatic nerve defect in rat. The objective of this study was to find a convenient way to combine GDNF-loaded gelatin microspheres and bilayer collagen membrane via GelMA hydrogel for nerve tissue engineering.

A combination of scanning electron microscopy (SEM), electrophysiological assessment, histology of regenerated nerves for hematoxylin-eosin (HE) staining, Loyez staining, and transmission electron microscopy (TEM) of all regenerated nerves was conducted to study axonal regeneration and functional recovery.

\section{Materials and methods Materials}

Type B gelatin B, 1-ethyl-3-(3-dimethyl aminopropyl) carbodiimide hydrochloride (EDC), tetramethylethylenediamine, ammonium persulfate, and methacrylic anhydride were purchased from Sigma. Recombinant rat GDNF was obtained from PeproTech. Acetone, petroleum ether, and polyoxymethylene were common laboratory analytical reagents from Sinopharm Chemical Reagent.

\section{GelMA hydrogels synthesis}

GelMA hydrogels were prepared by following previous reports. ${ }^{21,22}$ Briefly, $15 \%(\mathrm{w} / \mathrm{v})$ gelatin was dissolved in stirred distilled water $\left(\mathrm{dH}_{2} \mathrm{O}\right)$ at $50^{\circ} \mathrm{C}$ for 1 hour. 
Methacrylic anhydride was added dropwise to a final volume ratio of 1:400 methacrylic anhydride:aqueous solution of gelatin. The solution was then stirred at $50^{\circ} \mathrm{C}$ for 1 hour. The resulting mixture was dialyzed (12 $\mathrm{kDa}$ molecular weight cutoff) for 3 days against $\mathrm{dH}_{2} \mathrm{O}$ with frequent water replacement every 8 hours. The dialyzed solution was lyophilized, and the resulting GelMA was stored at $-20^{\circ} \mathrm{C}$ until use.

\section{Gelatin microsphere synthesis and characterization}

Gelatin microspheres were prepared according to a previously established method with slight modifications. ${ }^{18}$ Briefly, $10 \mathrm{~mL}$ of an aqueous solution of $10 \%(\mathrm{w} / \mathrm{v})$ Type B gelatin (Sigma) was preheated to $45^{\circ} \mathrm{C}$, and added dropwise into $50 \mathrm{~mL}$ of liquid paraffin containing $1 \%$ span $80 .{ }^{16}$ The two phases were emulsified for 15 minutes using magnetic stirrer. The emulsion was then rapidly cooled to $4^{\circ} \mathrm{C}$ in an ice bath, and stirring was continued to allow the spontaneous gelation of the gelatin aqueous solution. After 30 minutes, $50 \mathrm{~mL}$ of chilled acetone $\left(4^{\circ} \mathrm{C}\right)$ was added to the stirring solution. After 1 hour, gelatin microspheres were formed in the aqueous phase. The gelatin microspheres were then rinsed in acetone several times to eliminate the remaining oil on their surfaces, and then air dried.

The microspheres $(500 \mathrm{mg}$ ) were suspended in $25 \mathrm{~mL}$ of acetone:water (8:2) containing $25 \mathrm{mM}$ of EDC and stirred using magnetic stirrer for 2 hours at $4^{\circ} \mathrm{C}$, and then, at room temperature, stirring was continued for 10 hours to further crosslinking. Crosslinked microspheres were collected by filtration $(<25 \mu \mathrm{m})$, washed three times with ultrapure deionized water $\left(\mathrm{dH}_{2} \mathrm{O}\right)$, and lyophilized.

The morphology of gelatin microspheres was observed by field-emission SEM (LEO 1530). The samples were mounted on copper stubs using a double-side adhesive tape and sputter coated with gold.

\section{GDNF-loaded microspheres and in vitro release}

Growth factor-loaded microspheres were prepared by a method of adsorption according to established methods. ${ }^{19}$ Briefly, aqueous GDNF solutions at $\mathrm{pH} 7.4$ were dropped onto the crosslinked microspheres $(10 \mathrm{mg})$ at a volume of $2 \mu \mathrm{L}$ of aqueous GDNF solutions $(8 \mu \mathrm{g} / \mathrm{mL})$ per milligram of microspheres and incubated at $4^{\circ} \mathrm{C}$ for 20 hours before freezedrying. At $\mathrm{pH} 7.4$, complexation occurs between positively charged GDNF ( $\mathrm{pI}$ of 9.23) and negatively charged Type B gelatin ( $\mathrm{pI}$ of 4.7-5.2). To ensure complete sorption of GDNF by gelatin microspheres, ${ }^{24}$ volume of GDNF-containing solutions used was less than that required for complete swelling of gelatin microspheres (Figure 1B).

To determine growth factor release from crosslinked microspheres, $10 \mathrm{mg}$ of GDNF-loaded microspheres was

A

B

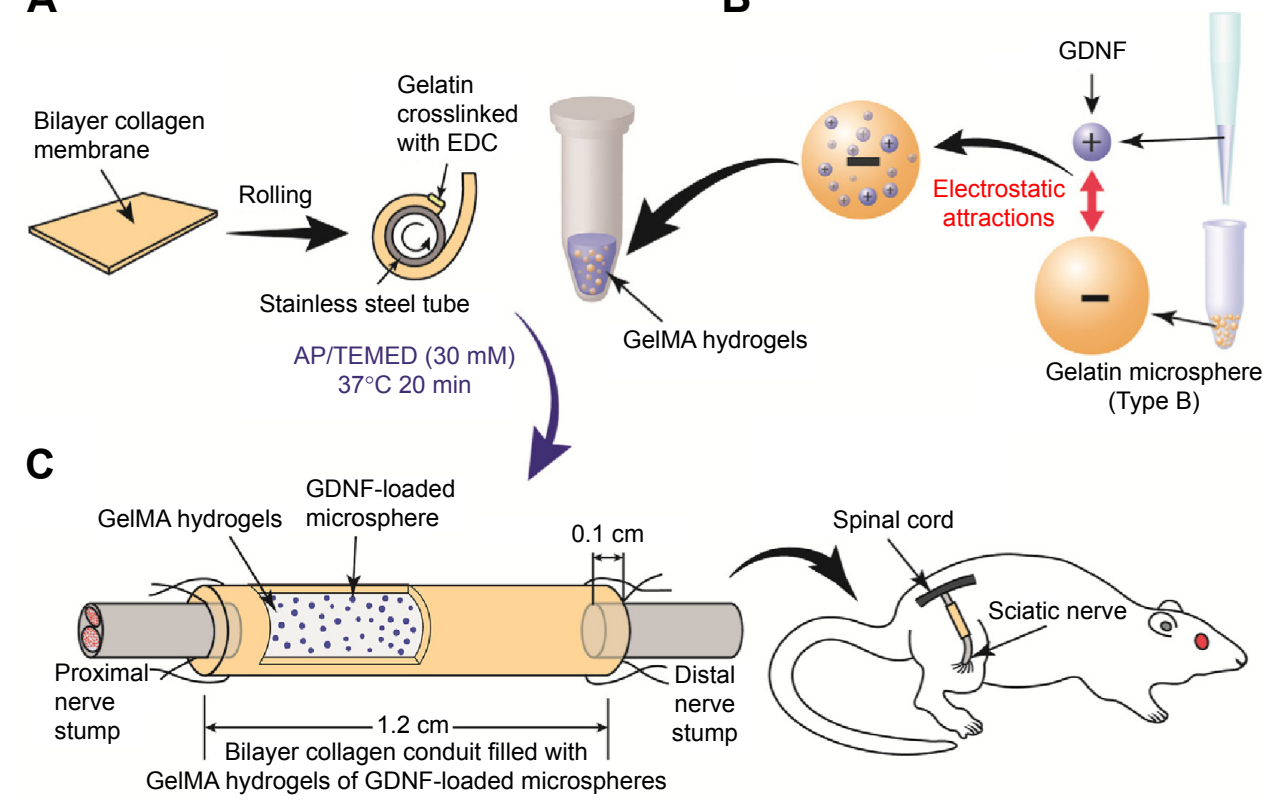

Figure I Schematic illustration of bilayer collagen conduit coated with gelatin-methacrylamide hydrogels of GDNF-loaded microspheres, bridging sciatic nerve in rats. Notes: (A) The membrane was rolled around a stainless steel tube, and the overlapping edges were crosslinked with gelatin to form a tubular structure. (B) Aqueous GDNF solutions were dropped into the crosslinked microspheres. GDNF-loaded microspheres were then mixed with GelMA at $37^{\circ} \mathrm{C}$. After polymerization, the gel was seeded into the inside layer of conduit. (C) A tube with a length of $12 \mathrm{~mm}$ was cut for implantation to bridge a I cm gap of sciatic nerve in rat with both ends entering the conduit by I mm. Abbreviations: GDNF, glial cell-line derived neurotrophic factor; GelMA, gelatin-methacrylamide; EDC, I-ethyl-3-(3-dimethyl aminopropyl) carbodiimide hydrochloride; $\mathrm{AP}$, ammonium persulfate; TEMED, tetramethylethylenediamine. 
taken in a microcentrifuge tube containing $400 \mu \mathrm{L}$ of phosphate-buffered saline $(\mathrm{pH} 7.4)(\mathrm{n}=3)$ and placed on a rotary shaker at $40 \mathrm{rpm}$ and $37^{\circ} \mathrm{C}$ for a month. At various points over a period of 32 days, samples were centrifuged, the supernatant was collected, and fresh release medium was added. In 32 days, all the supernatants were quantified by an enzyme-linked immunosorbent assay (Boster, Wuhan, People's Republic of China) for rat GDNF according to the manufacturer's instructions. The absorbance was read at $450 \mathrm{~nm}$ with an optical subtraction at $540 \mathrm{~nm}$ using a multi-well plate spectrophotometer, and sample concentrations were calculated from a standard curve of known GDNF concentrations.

\section{Collagen nerve conduit combined with GDNF-loaded microsphere fabrication}

Bilayer collagen membrane (Bio-Gide) was cut to the appropriate size. Collagen membrane and GelMA hydrogels were UV sterilized for 1 hour. Then, the membrane was rolled around a stainless steel tube, and the edge was fixed with gelatin hydrogen (10\%) and crosslinked with EDC (200 mM) for 10 hours to form a tubular structure (Figure 1A). Collagen nerve conduit was washed three times with $\mathrm{dH}_{2} \mathrm{O}$, and then lyophilized. A volume of $10 \mathrm{mg}$ GDNF-loaded microspheres was mixed with $110 \mu \mathrm{L}$ of $15 \%$ GelMA hydrogels, which was preheated to $37^{\circ} \mathrm{C}$. Then, $5 \mu \mathrm{L}$ of tetramethylethylenediamine $(800 \mathrm{mM})$ and $5 \mu \mathrm{L}$ of ammonium persulfate $(800 \mathrm{mM})$ were added into the mixture separately. Then, $130 \mu \mathrm{L}$ of final mixture was seeded into the inside layer of conduit, and the conduit was rotated around longitudinal axis gently for uniform distribution of GelMA hydrogel, then incubated at $37^{\circ} \mathrm{C}$ for at least 20 minutes before use. All these procedures were performed under sterile conditions.

\section{Surgical procedure}

All the animals used in this study were purchased from Nanjing Medical University Experimental Animal Center. All animal handling procedures were performed according to the Guide for the Care and Use of Laboratory Animals of the National Institutes of Health and were approved by Animal Care and Use Committee of Nanjing Medical University/Institutional Animal Care and Use Committee (NJMU/IACUC). The animals had free access to standard food and water and were housed on a 12 hour light/dark cycle at $22^{\circ} \mathrm{C}$ room temperature in the animal facilities at Nanjing Medical University.

Male Sprague Dawley rats ( $n=21)$, weighing $~ 200-250$ g, were randomly allocated into four groups: BLANK group $(n=3)$, nerve autograft (AG) group $(n=6)$, collagen tube
(CT) group $(n=6)$, and collagen tube filled with GDNFloaded microspheres $(\mathrm{GM}+\mathrm{CT})$ group $(n=6)$. Each rat was anesthetized by an intraperitoneal injection of $10 \%$ chloral hydrate $(300 \mathrm{~mL} / 100 \mathrm{~g}$ body weight). The thigh areas on right sides were shaved, and povidone-iodine was applied. The operative instruments were autoclaved and packaged under sterile conditions, and disposable knife blades, syringes, and drapes were used. The surgical procedures were performed under sterile conditions. Right sciatic nerve was chosen as the experimental side in each animal, and left sciatic serve as normal nerve group. Then, a skin incision was made to expose the sciatic nerve, and the underlying muscles were split in the right thigh. In the nerve AG group, a $10 \mathrm{~mm}$ nerve was resected, and then reversed $180^{\circ}$ and reimplanted bridging the gap. In the BLANK group, we left the $10 \mathrm{~mm}$ gap empty after resection. In the CT group and GM + CT group, a 7-8 mm long piece of nerve was removed, leaving a $1 \mathrm{~cm}$ gap following retraction of the nerve ends. Then, $1 \mathrm{~cm}$ gap was bridged with collagen tube or collagen tube with GDNF-loaded microspheres. The conduit was sutured to both the proximal and distal nerve stumps with 7-0 nylon sutures (Figure 1C). In all animals, the skin was closed with 5-0 sutures.

\section{Electrophysiological assessment of CMAPs}

Each animal underwent electrophysiological testing at 20 weeks postoperation. After anesthesia, the electrophysiologic tests were performed by placing stimulation electrode on the sciatic nerve trunk and recording electrodes on the mid of gastrocnemius. The nerve stimulation parameters included a duration of $0.04 \mathrm{~ms}$ and intensity of $20 \mathrm{~mA}$ to elicit compound-evoked muscle action potential (CMAP). The distance between the stimulating electrode and the musclerecording electrode was $2 \mathrm{~cm}$. Latency (LAT) and CMAPs were also recorded across the reconstructed defects in the postoperative period. The LAT was measured in milliseconds from stimulation site to start of the response. The amplitude (AMP) of CMAPs was also measured in millivolts.

\section{Histology and histomorphometry}

After sacrifice, the anastomotic sites (grafts or conduits plus an $\sim 5 \mathrm{~mm}$ portion from the proximal and distal nerve stumps) were harvested for histological examination. A 5-0 suture was inserted into the proximal end of the nerve at the time of harvest to provide anatomical orientation during analysis. Nerves were fixed in $10 \%$ neutral buffered formalin for 48 hours. The section of middle parts of the regenerated nerves was stained with $\mathrm{HE}$ and observed under a light microscope equipped with a camera. 


\section{TEM sample preparation}

Following harvesting, the specimens were fixed in $2.5 \%$ glutaraldehyde. After fixation in 1\% osmium tetroxide and dehydration in ethanol, the specimens were embedded in Agar 100. Ultrathin sections of $50 \mathrm{~nm}$ thickness were prepared using an ultramicrotome, placed on copper grids, stained with lead citrate, and examined by TEM. Quantitative morphometry was performed measuring the mean diameter of axon, the myelination thickness, density of myelinated axons (the number of myelinated axons per 1,000 $\mu^{2}$ ), and G-ratio (axon-to-fiber diameter) in every section.

\section{Statistical analysis}

All quantitative results are presented as mean \pm standard deviations. The data were evaluated using single-factor analysis of variance and Tukey's test for simultaneous paired comparisons if conditions of normality and equal variance were met. Data of mean diameter of myelinated nerve fibers and thickness of nerve myelin were tested assuming unequal variance, so groups were compared with nonparametric tests (Kruskal-Wallis test). A value of $P<0.05$ was considered statistically significant.

\section{Results and discussion}

In this study, we first fabricated collagen tube with commercial bilayer collagen membrane (Bio-Gide). In order to improve the nerve growth, we synthesized GDNF-loaded microspheres. The microspheres were coated with GelMA hydrogel onto the inner conduit. The tube with microspheres formed a novel biodegradable nerve conduit to bridge a $10 \mathrm{~mm}$ long sciatic nerve defect in rat.

When nerve injury occurs, the proximal stump of the peripheral nerve begins with a scenario of regeneration process, while the distal stump undergoes degeneration. After injury, the distal nerve stump and target tissues are chronically denervated along with nerve and target tissues atrophy. SCs play a key role in nerve regeneration. SCs maintain tubes of basal lamina which forms bands of Büngner; therefore, they play a significant guiding role in the linear growth of regenerated axons. Meanwhile, Wallerian degeneration occurs in the distal stump, and SCs and macrophages interact to remove debris, specifically myelin and the damaged axon, for reinnervation. ${ }^{11}$ Together, these create a healthy microenvironment for PNS regeneration. So tissueengineering methods in different forms have been reported to regenerate nerve and achieved good results via enhancing proliferation of SCs or feeding SCs directly. ${ }^{11,27}$

\section{GDNF-loaded microspheres}

The GDNF-loaded gelatin microspheres were observed by scanning electron microscope (Figure 2A). Gelatin microsphere had a smooth surface and good dispersion. The mean diameter of GDNF-loaded microspheres was $3.91 \pm 1.87 \mu \mathrm{m}$ in normal distribution.
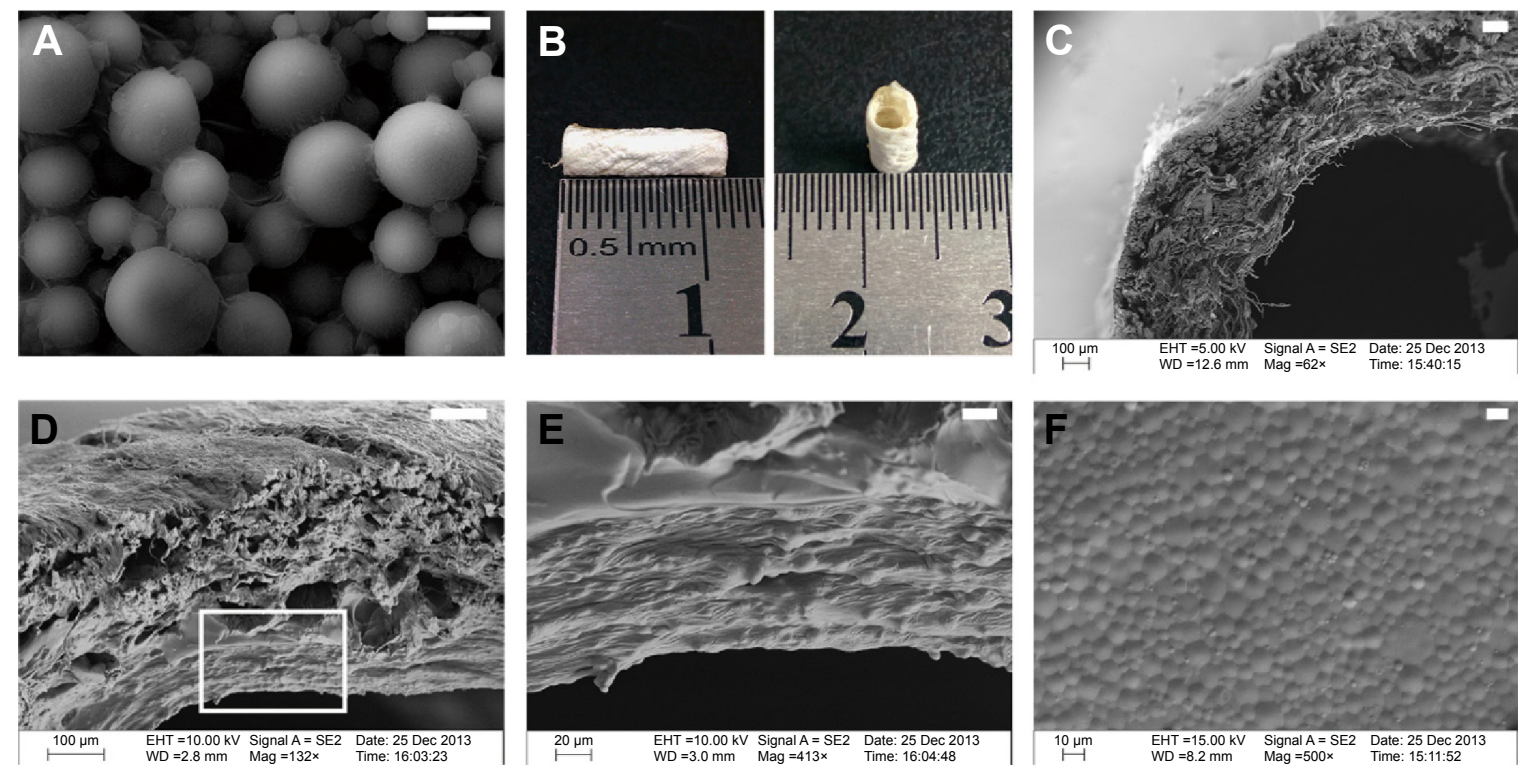

Figure 2 Macroscopic and microscopic morphology of bilayer collagen conduit filled with GelMA hydrogels of GDNF-loaded microspheres.

Notes: (A) GDNF-loaded gelatin microspheres were observed by scanning electron microscope. (B) Macroscopic morphology of bilayer collagen conduit fabricated with bilayer collagen membrane. (C) Bilayer collagen conduit showing the layered structures in SEM. (D-F) SEM observations of bilayer collagen conduit filled with GelMA hydrogels of GDNF-loaded microspheres. (E) Magnified views of the square area in (D). (F) The surface morphology of GelMA hydrogel mixed with GDNF-loaded gelatin microspheres (bar in $(\mathbf{A})=4 \mu \mathrm{m}$; bar in $(\mathbf{C})=100 \mu \mathrm{m}$; bar in $(\mathbf{D})=100 \mu \mathrm{m}$; bar in $(\mathbf{E})=20 \mu \mathrm{m}$; bar in $(\mathbf{F})=10 \mu \mathrm{m})$.

Abbreviations: GelMA, gelatin-methacrylamide; GDNF, glial cell-line derived neurotrophic factor; SEM, scanning electron microscopy. 


\section{Characterizations of bilayer collagen conduit filled with GelMA hydrogels of GDNF-loaded microspheres}

An ideal nerve repair material should have the following properties: biocompatibility to prevent chronic inflammation, with no adverse impact on host; mechanism to provide appropriate room for nerve regeneration; permeability to prevent scar tissue from invading and permit the permeation of nutrients and oxygen; biodegradability to get degraded completely in a reasonable period of time; and production of nontoxic degradation products.

In this study, collagen conduit is $-1.2 \mathrm{~cm}$ in length, $3.5 \mathrm{~mm}$ in outer diameter, and $2 \mathrm{~mm}$ in inner diameter (Figure 2B). The size of collagen conduit is suitable for bridging $1 \mathrm{~cm}$ gap of sciatic nerve in rat.

SEM images showed bilayer structure of collagen conduit (Figure 2C). The inner layer consisted of loosely arranged collagen fibers, while the outer layer of the membrane contained dense and tight fibers. This structure can prevent ingrowth of soft tissue into the lumen, and promote cell attachment and exchange of nutrients and metabolites. Figure 2D shows that layer of GelMA hydrogel mixed with GDNF-loaded gelatin microspheres was coated on the inside layer of collagen conduit. GDNF-loaded gelatin microspheres could be observed in the transverse sections of GelMA hydrogel (Figure 2E). SEM image of the surface morphology of GelMA hydrogel mixed with GDNF-loaded gelatin microspheres showed that gelatin microsphere were densely arranged and distributed evenly on the surface (Figure 2F).

\section{GDNF release study in vitro}

In a neutral condition ( $\mathrm{pH} 7.4$ ) (Figure 3A), we tested Type B and Type A gelatin microspheres. We observed the initial burst release of GDNF-loaded Type B gelatin microspheres on the first day, and the release amounted to $15.0 \% \pm 2.1 \%$. Then, burst release reduced significantly in 2-3 days, amounting to $3.0 \% \pm 1.4 \%$. Three days later, the release slowed down in a linear manner (average release of $0.1 \% \mathrm{GDNF} /$ day for 4-32 days). We used Type A gelatin microspheres as control microsphere (Figure 3B). The initial burst release of Type A gelatin microspheres was significantly higher than Type $\mathrm{B}$, and the release amounted up to $98.9 \% \pm 0.9 \%$ on the first day.
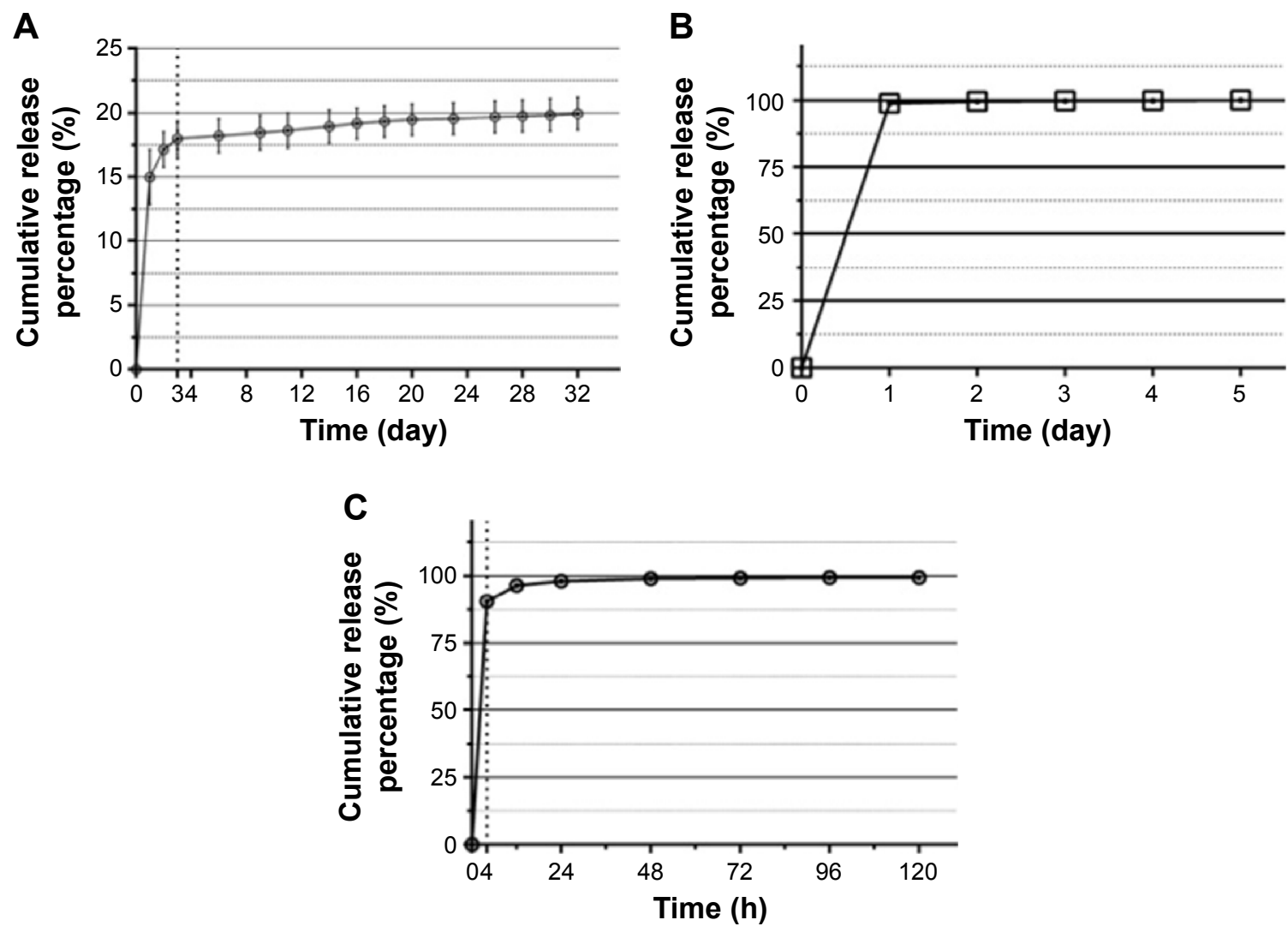

Figure 3 The cumulative release curves of GDNF measured with ELISA.

Notes: (A) The cumulative release amount of GDNF from Type B gelatin microspheres in a neutral condition (pH 7.4) for 32 days. (B) In vitro release of GDNF from Type A gelatin microspheres in a neutral condition ( $\mathrm{pH} 7.4$ ) for 5 days. (C) The cumulative release percentage of GDNF from Type B gelatin microspheres in an acidic condition $(\mathrm{pH} 2.5)$ for 5 days.

Abbreviations: GDNF, glial cell-line derived neurotrophic factor; ELISA, enzyme-linked immunosorbent assay. 
In an acidic condition ( $\mathrm{pH} 2.5$ ), as shown in Figure 3C, Type B gelatin microspheres and GDNF were having the same charge. The initial release percentage of GDNF was up to $91.2 \% \pm 0.9 \%$ within 4 hours. The cumulative release percentage of GDNF was $98.3 \% \pm 0.2 \%$ at 24 hours and $99.2 \% \pm 0.2 \%$ at 48 hours.

GDNF can support the survival of dopaminergic and motor neurons, and is one of the most protective factors for motor neurons. And many studies have demonstrated that the effects of exogenous GDNF on motor axonal are not the more the better. Local delivery of low-dose GDNF can increase axonal regeneration effectively, while high-dose GDNF will not further enhance the effect, and even cause a worse outcome. ${ }^{28,29}$ Mills et al investigated the effects of GDNF delivered directly to dorsal root ganglion cell bodies in vivo. ${ }^{28}$ They reported that low-dose GDNF $(0.2 \mathrm{ng} / \mathrm{day} /$ 7 days) increased axonal regeneration greater than high-dose GDNF (2.0 or $20 \mathrm{ng} /$ day/7 days). So local delivery of lowdose GDNF is appropriate for PNS regeneration.

We developed GDNF-loaded gelatin microspheres for sustained release. GDNF-loaded microspheres were prepared by complexation between positively charged GDNF (pI of 9.23) and negatively charged Type B gelatin ( $\mathrm{pI}$ of 4.7-5.2) in a neutral condition. The complexation provides the following advantages: binding of microspheres and growth factor under mild conditions without significant effects on the activity of growth factor and drug loading efficiency up to $\sim 100 \%$. Theoretically, in systems in which controlled release occurs via electrostatic attractions, there are two release mechanisms: carrier degradation (main factor) and ionic depolymerization due to environmental changes. ${ }^{30}$ So the growth factors undergo sustained release as the gelatin degrades. ${ }^{19,24}$ In vitro release studies with gelatin microspheres exhibited minimal initial burst release during the first 3 days $(18.0 \% \pm 1.3 \%)$ in neutral release environment ( $\mathrm{pH} 7$ ) (Figure 3A). Then, the release slowed down in a linear manner (average release of $78 \mathrm{pg}$ GDNF/day for 4-32 days), and the average environmental concentration of GDNF was $195 \mathrm{pg} / \mathrm{mL}$. A significantly higher initial burst release was observed in the first 3 days when we used Type A gelatin microspheres as control microspheres. However, in an acid condition ( $\mathrm{pH} 2.5$ ) (Figure 3C), gelatin microspheres release rate was up to $91.2 \% \pm 0.9 \%$ within 4 hours. This may be due to charge change from negative to positive of Type B gelatin in acidic condition, which leads to its mutually exclusive status. This indicates that GDNF and Type B gelatin microspheres can form a stable controlled-release system via electrostatic attraction.

\section{Electrophysiological assessment}

Electrophysiological studies were performed to evaluate the functional reinnervation through NGS. The CMAPs of BLANK, GM-CT, CT, and AG group were compared with the normal group at 20 weeks postoperation (Figure 4A-D).
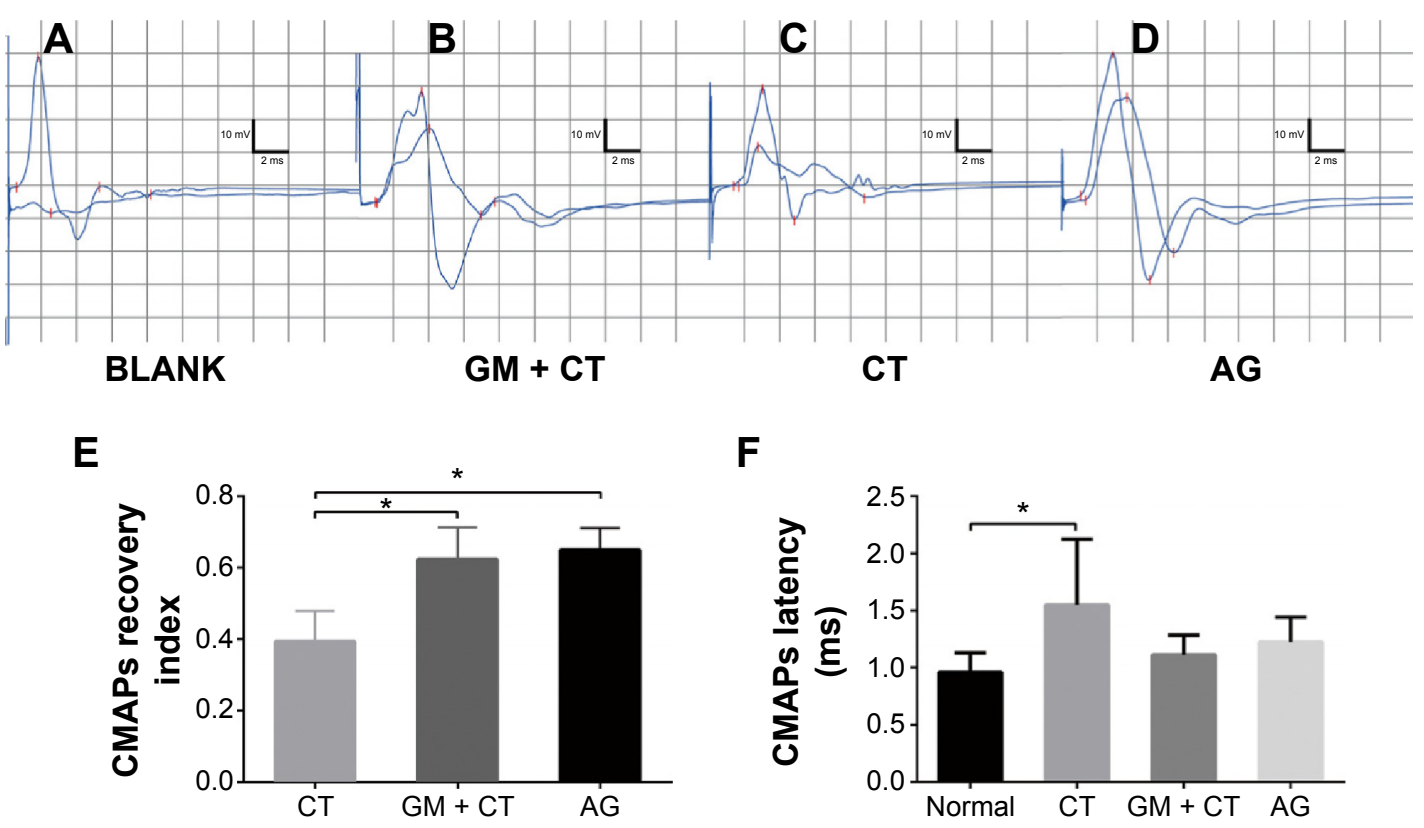

Figure 4 CMAP detection of gastrocnemius muscle at 20 weeks' postoperation.

Notes: Representative data of the (A) BLANK group, (B) GM + CT group, (C) the CT group, and (D) AG group. Data comparison of CMAP recovery index and latency is shown in $(\mathbf{E})$ and $(\mathbf{F})(\mathrm{n}=6)$. $* \mathrm{P}<0.05$.

Abbreviations: CMAP, compound-evoked muscle action potential; GM + CT, collagen tube filled with GDNF-loaded microspheres; CT, collagen tube; AG, autograft; GDNF, glial cell-line derived neurotrophic factor. 
LAT and CMAPs recovery index can serve as the important parameters for evaluating the function of the regenerated nerve. We could not evoke CMAP in BLANK group. The action potentials of regenerated nerves were similar to the normal nerves, with a lower AMP and longer LAT. The CMAPs recovery index showed that GM + CT group was not significantly different from AG group $(P>0.5)$; $\mathrm{CT}$ group had significantly fewer CMAPs recovery index than AG and GM + CT group $(P<0.05)$ (Figure 4E). The CMAPs LAT showed that only CT group had significantly longer LAT than the normal group. And there were no statistically significant differences between other groups $(P>0.05)$ (Figure 4E and F).

\section{Nerve histology}

No regenerated nerve bridged the $1 \mathrm{~cm}$ defect in BLANK group, and neuroma was formed at the end of the proximal stump. Except in the BLANK group, the nerve regeneration was successful, and the structure of NGSs had degraded completely at 20 weeks postoperation, and no inflammatory signs or adverse tissue reaction were seen in $\mathrm{GM}+\mathrm{CT}, \mathrm{CT}$, and $\mathrm{AG}$ groups (Figure 5C-E). Collagen, as a key component of nature extracellular matrix, can be degraded by collagenase in vivo, and the degradation product was nontoxic. Complete degradation of Bio-Gide was reported 3-4 months following implantation in animal experiment. ${ }^{31}$

HE staining was used to assess regenerated nerve morphology. Nerve regeneration was observed in the transverse sections of midsection of regeneration nerve in all three groups. In AG and GM + CT group, apparently ordered structures could be observed, and the arrangements of nerve fibers appeared more compact and regular compared with CT group (Figure 6A1-D1). The nerve cross-sectional area is observed in descending order: normal, $\mathrm{AG}, \mathrm{GM}+\mathrm{CT}$, and CT group $(P<0.05)$ (Figure $6 \mathrm{~F})$. In $\mathrm{CT}$ group, compared with normal and AG groups, lots of new blood vessels and connective tissue were found around the nerve fiber.
Longitudinal histology of regenerated nerves for $\mathrm{HE}$ staining showed that nerve fibers in AG and GM + CT group were almost regenerated completely and arranged in neat pattern along the long axis, while the nerve fiber of CT group exhibited disordered arrangement (Figure 6A2-D2). Many cell nuclei of SCs can be observed in regenerated nerve unlike the normal nerve.

In BLANK group, the nerve could not regenerate to form normal nerve structure (Figure 6E1 and E2). Furthermore, we observed neuroma formed at the end of the proximal stump (Figure 6E3). Due to the absence of regenerated nerve bridging the defect gap, we could not evoke CMAP in electrophysiological assessment (Figure 4A).

\section{Transmission electron microscopy}

Figure $7 \mathrm{~A}-\mathrm{C}$ shows transverse sections of the regenerated nerve tissue at 20 weeks postoperation observed using TEM. Since nerve did not regenerate in BLANK group, TEM observation did not include the BLANK group.

We could observe the myelinated axons using the TEM in all groups. In GM + CT group (Figure 7B), most myelinated nerves were matured and densely arranged with a uniform structure of myelin which was similar to the AG group but with thinner myelin sheath and small diameters of axons. However, there were many nonmyelinated nerves and SCs observed in the CT group. Quantitative analysis showed that the mean thinness of the myelin sheath in $A G$ and $\mathrm{GM}+\mathrm{CT}$ group was significantly higher than that in the CT group $(P<0.05)$ (Figure 7D). The mean diameter of myelinated axons in AG and GM + CT group was significantly larger than that in the CT group $(P<0.05)$ (Figure $7 \mathrm{E})$. The myelinated fiber number (per 1,000 $\mu^{2}$ ) in AG and $\mathrm{GM}+\mathrm{CT}$ group was significantly larger than that in the CT group $(P<0.05)$ (Figure $7 \mathrm{~F})$. And these parameters were similar in AG and GM + CT groups $(P>0.05)$ (Figure 7D-F). In addition, G-ratio, a highly reliable ratio for assessing axonal myelination, was found to be statistically equivalent

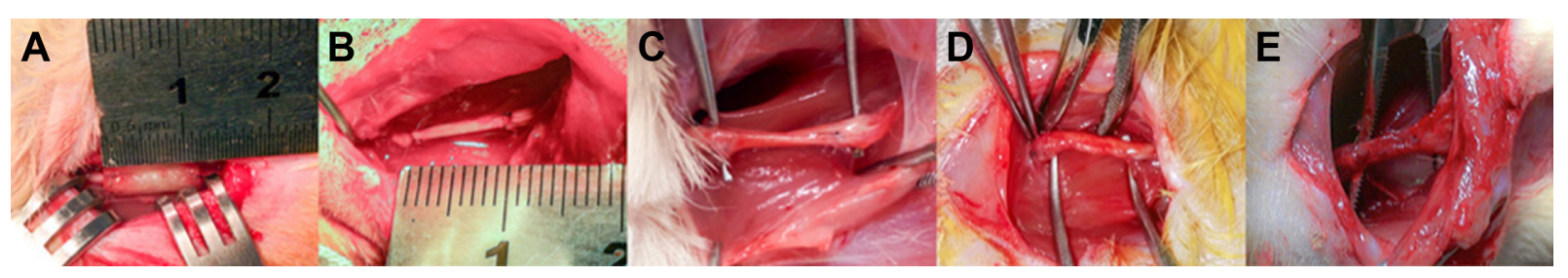

Figure 5 Experimental model.

Notes: Surgical implantation of (A) collagen tube or collagen tube with GDNF-loaded microspheres and (B) autograft nerve to bridge I cm defect of sciatic nerve. The regenerated nerve of (C) GM + CT group, (D) CT group, and (E) AG group at 20 weeks postoperation.

Abbreviations: GDNF, glial cell-line derived neurotrophic factor; GM + CT, collagen tube filled with GDNF-loaded microspheres; CT, collagen tube; AG, autograft. 

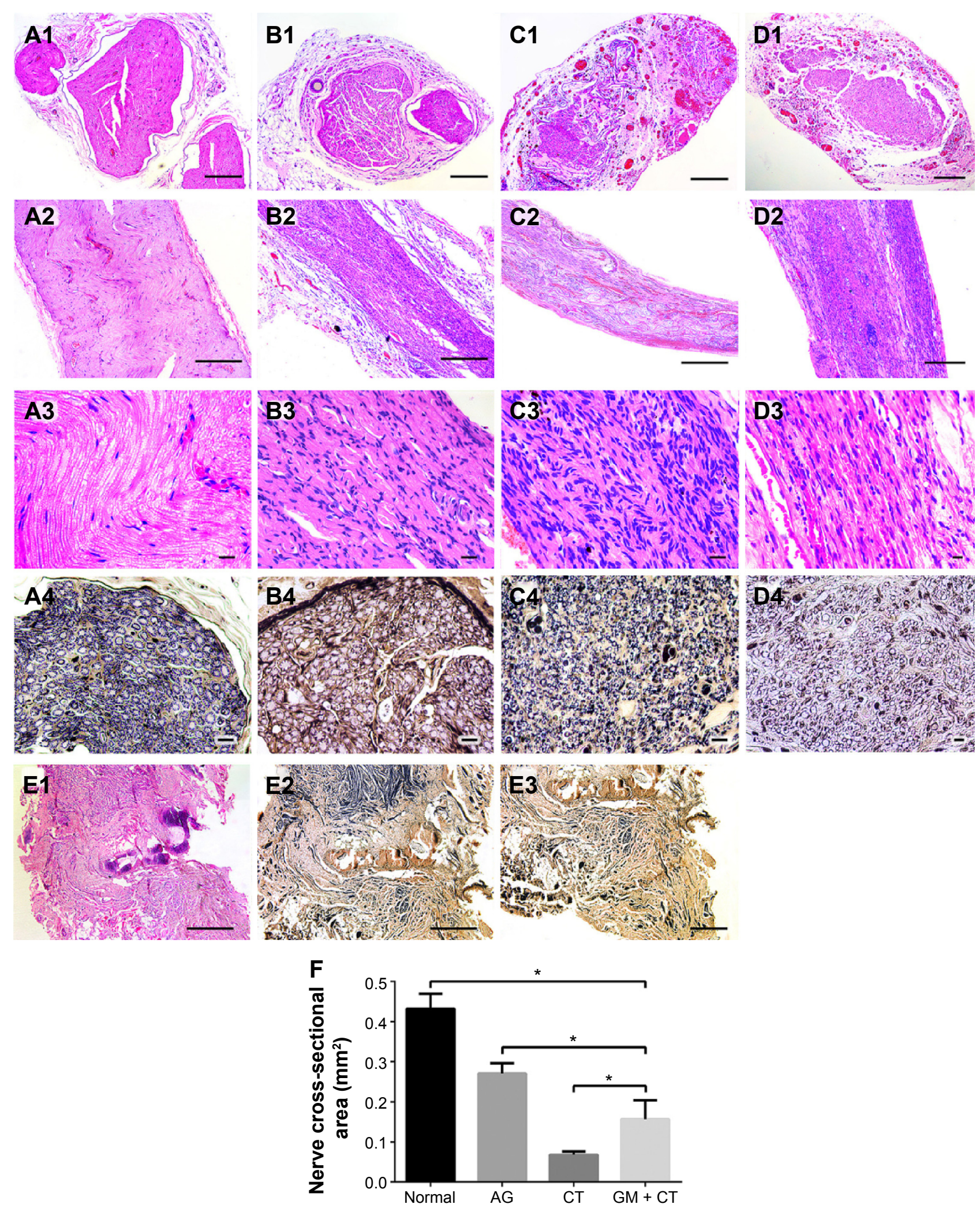

Figure 6 Histology of regenerated nerves for HE staining and Loyez staining under light microscopy at 20 weeks postoperation.

Notes: (A) Normal group. (B) AG group. (C) CT group. (D) GM + CT group. (E) BLANK group. (AI-DI) HE staining of transverse sections of midsection of regeneration nerve. (A2-D2, A3-D3, and EI) HE staining of longitudinal histology of regenerated nerves. (A4-D4 and E2, E3) Loyez staining. (E3) Neuroma formed at the end of the proximal stump (bar in [AI-DI] and [E3] $=200 \mu \mathrm{m}$; bar in [A2-D2] and [EI and E2] $=500 \mu \mathrm{m}$; bar in [A3-D3] $=20 \mu \mathrm{m}$; bar in [A4-D4] $=10 \mu \mathrm{m}$ ). (F) Statistical analysis of the nerve cross-sectional area $* P<0.05$.

Abbreviations: $\mathrm{HE}$, hematoxylin-eosin; $\mathrm{AG}$, autograft; $\mathrm{CT}$, collagen tube; $\mathrm{GM}+\mathrm{CT}$, collagen tube filled with GDNF-loaded microspheres; GDNF, glial cell-line derived neurotrophic factor. 


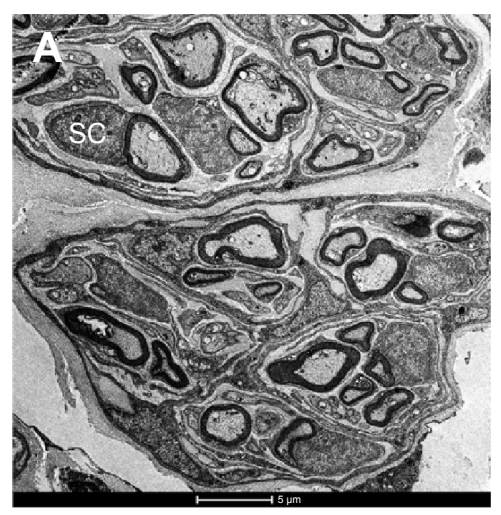

D
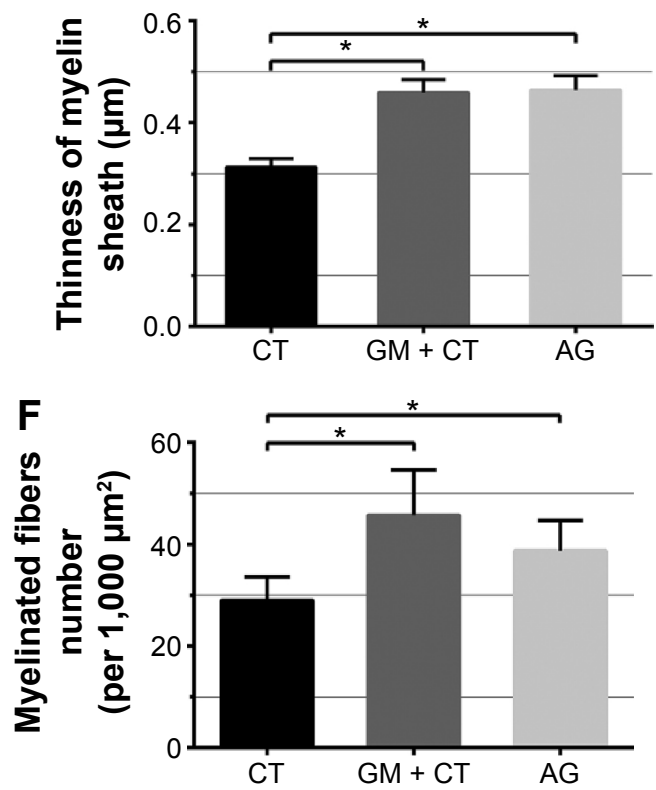
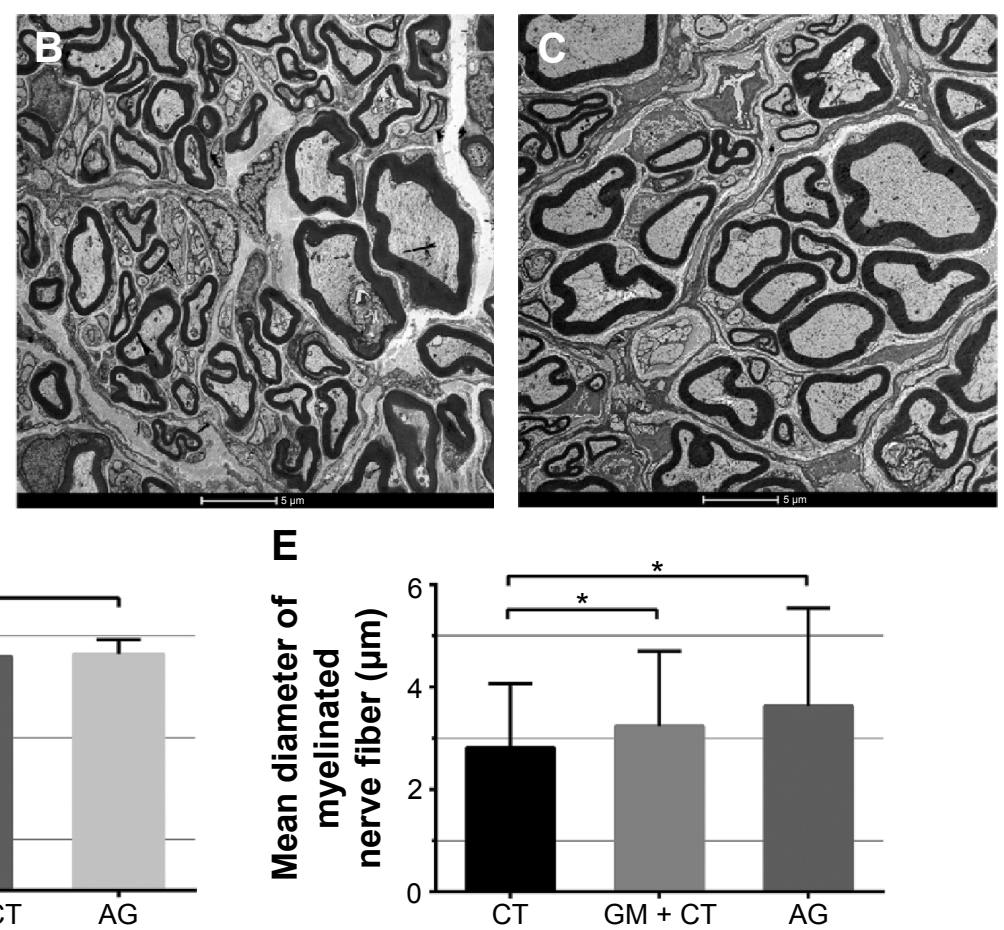

G

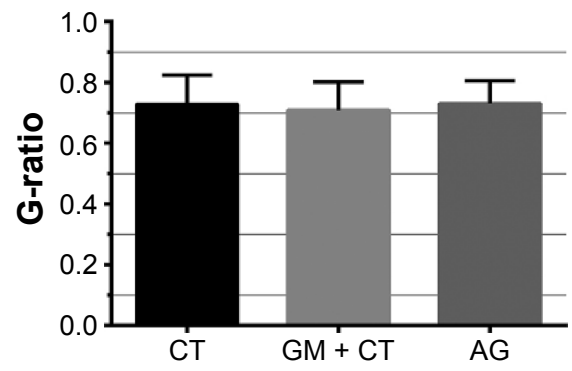

Figure 7 Histologic observation and morphometric analysis of regenerated nerves under transmission electron microscopy.

Notes: (A-C) Transmission electron microscopy of transverse sections of the regenerated nerve tissue at 20 weeks postoperation and (D) statistical analysis of the myelin sheath thickness, (E) diameters of myelinated nerves, (F) myelinated fibers number (per I,000 $\mu \mathrm{m}^{2}$ ), and $(\mathbf{G}) \mathrm{G}$-ratio for each group. $\mathrm{n}=3$. ${ }^{*} \mathrm{P}<0.05$. $(\mathbf{A}) \mathrm{CT}$ group. (B) $\mathrm{GM}+$ CT group. (C) AG group. Scale bar $=5 \mu \mathrm{m}$.

Abbreviations: CT, collagen tube; GM + CT, collagen tube filled with GDNF-loaded microspheres; AG, autograft; GDNF, glial cell-line derived neurotrophic factor; SC, Schwann cells.

in all groups: CT $(0.73 \pm 0.10), \mathrm{GM}+\mathrm{CT}(0.71 \pm 0.09)$, and

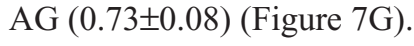

\section{Histological assessment of target muscle}

To evaluate the atrophy of rat gastrocnemius muscle, due to denervated status, gastrocnemius muscle of the four groups was stained with HE (Figure 8A-D). Gastrocnemius muscles suffered from degeneration and presented with a smaller diameter of muscle fibers, more fibrous connective tissue, and blood vessels. Gastrocnemius of AG, CT, and GM + CT group had remarkable atrophy compared to normal group, but the muscle fibers atrophy was found to be most obvious in the CT group. The muscle wet weight ratio shows no significant difference between CT and GM + CT group, but it was less than that of AG group (Figure 8E). The average diameter of muscle fibers of AG and GM + CT group was smaller than the normal group but larger than the CT group (Figure 8F).

For nerve regeneration, the main obstacle is to restore the function of the target organ. In this study, to evaluate axonal regeneration and functional recovery, electrophysiological assessment and histomorphometry analysis were performed. Theoretically, the AMP of CMAP can estimate the number of axons reinnervating in distal target muscle fibers, while the diameter of axons and the thickness of myelin sheath correlate with the velocity of conduction. From the electrophysiological point of view, each nerve regeneration group could evoke CMAPs with varying degrees, and function of target organ could be recovered. However, the functional recovery was not perfect. The CMAPs recovery index in AG and GM + CT group hit 65\%, while CT group seemed 

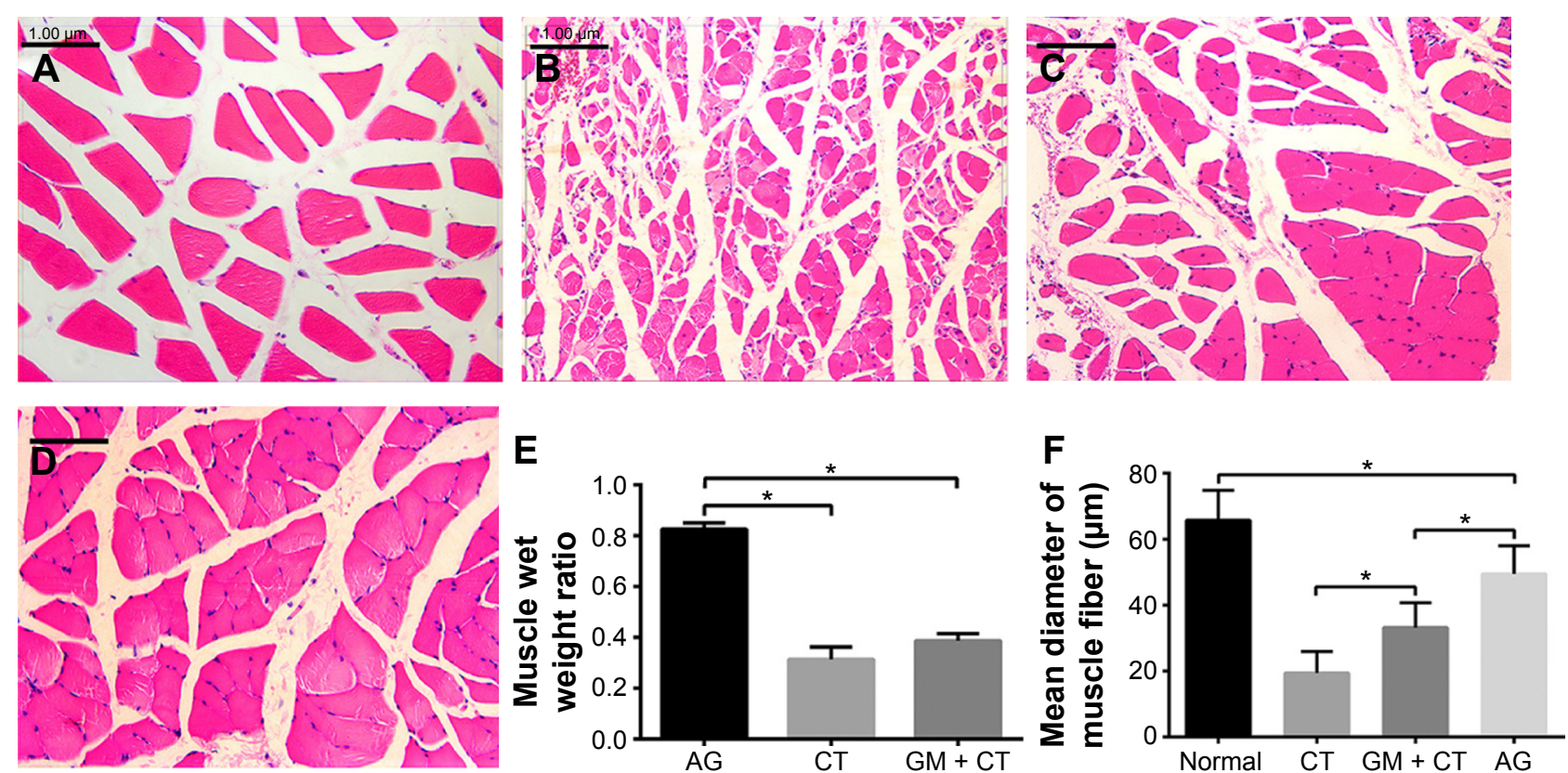

Figure 8 Gastrocnemius muscle histology at 20 weeks postoperation.

Notes: (A) Normal. (B) CT group. (C) GM + CT group. (D) AG group (scale bar $=100 \mu \mathrm{m}$ ). Statistical analysis of muscle wet weight ratio (E) and mean diameter of fiber (F). $* P<0.05$.

Abbreviations: CT, collagen tube; GM + CT, collagen tube filled with GDNF-loaded microspheres; AG, autograft; GDNF, glial cell-line derived neurotrophic factor.

worse (only 40\%). Gastrocnemius atrophy was significant in each group, and in the CT group, it was most evident. This is consistent with CMAP results. However, AG and GM + $\mathrm{CT}$ groups have more significant regeneration effects from the nerve histological point of view. Perhaps, this is because nerve recovery is not equivalent to the functional recovery of target organ. In mice, a critical window of $\sim 35$ days seems to exist, during which regenerating axons can reestablish connections with the distal muscles. ${ }^{32}$ However, the effective time of nerve regeneration is $4-8$ weeks in rodents. Once $\mathrm{SC}$ tubes deteriorate in the distal stump, the tissues are no longer receptive to innervation by new axons. Moreover, when reinnervation of remaining muscle finally occurs, neuromuscular synapses are able to form, but the muscle never fully recovers. ${ }^{1}$ So sometimes, even if the nerve regenerates, the function of target tissue is difficult to restore. For this reason, target organ is difficult to recover after nerve injuries in clinical practice. Thus, future research on the effect of nerve regeneration is needed, and target organ functional recovery should be paid more attention.

\section{Acknowledgments}

The authors gratefully acknowledge the support from Nature Science Foundation of Jiangsu Province (number BK2011844), the Six Talent Peak Project Foundation of Jiangsu Province (number WSW-021), and the Program for Developments of Innovative Research Team in the First
Affiliated Hospital with Nanjing Medical University (number IRT-015) funds, and NSERC discovery grant.

\section{Disclosure}

The authors report no conflicts of interest in this work.

\section{References}

1. Scheib J, Hoke A. Advances in peripheral nerve regeneration. Nature Reviews. Neurology. 2013;9(12):668-676.

2. Konofaos P, Ver Halen JP. Nerve repair by means of tubulization: past, present, future. Journal of Reconstructive Microsurgery. 2013;29(3): $149-164$.

3. Lundborg G. A 25-year perspective of peripheral nerve surgery: evolving neuroscientific concepts and clinical significance. The Journal of Hand Surgery. 2000;25(3):391-414.

4. Bozkurt A, Lassner F, O'Dey D, et al. The role of microstructured and interconnected pore channels in a collagen-based nerve guide on axonal regeneration in peripheral nerves. Biomaterials. 2012;33(5): 1363-1375.

5. Hsu SH, Kuo WC, Chen YT, et al. New nerve regeneration strategy combining laminin-coated chitosan conduits and stem cell therapy. Acta Biomaterialia. 2013;9(5):6606-6615.

6. Huang YC, Huang YY. Biomaterials and strategies for nerve regeneration. Artificial Organs. 2006;30(7):514-522.

7. Chamberlain LJ, Yannas IV, Arrizabalaga A, Hsu HP, Norregaard TV, Spector M. Early peripheral nerve healing in collagen and silicone tube implants: myofibroblasts and the cellular response. Biomaterials. 1998; 19(15):1393-1403.

8. Ma F, Xiao Z, Chen B, Hou X, Dai J, Xu R. Linear ordered collagen scaffolds loaded with collagen-binding basic fibroblast growth factor facilitate recovery of sciatic nerve injury in rats. Tissue Engineering. Part A. 2014;20(7-8):1253-1262.

9. Kozlovsky A, Aboodi G, Moses O, et al. Bio-degradation of a resorbable collagen membrane (Bio-Gide) applied in a double-layer technique in rats. Clinical Oral Implants Research. 2009;20(10):1116-1123. 
10. O'Brien FJ, Harley BA, Yannas IV, Gibson LJ. The effect of pore size on cell adhesion in collagen-GAG scaffolds. Biomaterials. 2005;26: 433-441.

11. Zhang YG, Sheng QS, Qi FY, et al. Schwann cell-seeded scaffold with longitudinally oriented micro-channels for reconstruction of sciatic nerve in rats. Journal of Materials Science: Materials in Medicine. 2013;24(7):1767-1780.

12. Yu W, Zhao W, Zhu C, et al. Sciatic nerve regeneration in rats by a promising electrospun collagen/poly(epsilon-caprolactone) nerve conduit with tailored degradation rate. BMC Neuroscience. 2011;12:68.

13. Niu Y, Chen KC, He T, Yu W, Huang S, Xu K. Scaffolds from block polyurethanes based on poly(varepsilon-caprolactone) (PCL) and poly(ethylene glycol) (PEG) for peripheral nerve regeneration. Biomaterials. 2014;35(14):4266-4277.

14. Tang X, Xue C, Wang Y, Ding F, Yang Y, Gu X. Bridging peripheral nerve defects with a tissue engineered nerve graft composed of an in vitro cultured nerve equivalent and a silk fibroin-based scaffold. Biomaterials. 2012;33(15):3860-3867.

15. Serban MA, Knight T, Payne RG, et al. Crosslinked gelatin microspheres with continuously tunable degradation profiles for renal tissue regeneration. Biotechnology and Applied Biochemistry. 2013;61(2):75-81.

16. Liang HC, Chang WH, Lin KJ, Sung HW. Genipin-crosslinked gelatin microspheres as a drug carrier for intramuscular administration: in vitro and in vivo studies. Journal of Biomedical Materials Research. Part A. 2003;65(2):271-282.

17. Chen YS, Chang JY, Cheng CY, Tsai FJ, Yao CH, Liu BS. An in vivo evaluation of a biodegradable genipin-cross-linked gelatin peripheral nerve guide conduit material. Biomaterials. 2005;26(18):3911-3918.

18. Wang H, Boerman OC, Sariibrahimoglu K, Li Y, Jansen JA, Leeuwenburgh SC. Comparison of micro- vs. nanostructured colloidal gelatin gels for sustained delivery of osteogenic proteins: bone morphogenetic protein-2 and alkaline phosphatase. Biomaterials. 2012;33(33): 8695-8703.

19. Kim S, Kang Y, Krueger CA, et al. Sequential delivery of BMP-2 and IGF-1 using a chitosan gel with gelatin microspheres enhances early osteoblastic differentiation. Acta Biomaterialia. 2012;8(5): 1768-1777.

20. Beigi MH, Ghasemi-Mobarakeh L, Prabhakaran MP, et al. In vivo integration of poly(epsilon-caprolactone)/gelatin nanofibrous nerve guide seeded with teeth derived stem cells for peripheral nerve regeneration. Journal of Biomedical Materials Research. Part A. 2014; $102(12): 4554-4567$
21. Schuurman W, Levett PA, Pot MW, et al. Gelatin-methacrylamide hydrogels as potential biomaterials for fabrication of tissue-engineered cartilage constructs. Macromolecular Bioscience. 2013;13(5): $551-561$.

22. Van Den Bulcke AI, Bogdanov B, De Rooze N, Schacht EH, Cornelissen M, Berghmans H. Structural and rheological properties of methacrylamide modified gelatin hydrogels. Biomacromolecules. 2000;1(1):31-38

23. Koshy ST, Ferrante TC, Lewin SA, Mooney DJ. Injectable, porous, and cell-responsive gelatin cryogels. Biomaterials. 2014;35(8): 2477-2487.

24. Patel ZS, Yamamoto M, Ueda H, Tabata Y, Mikos AG. Biodegradable gelatin microparticles as delivery systems for the controlled release of bone morphogenetic protein-2. Acta Biomaterialia. 2008;4(5): 1126-1138.

25. Madduri S, Feldman K, Tervoort T, Papaloizos M, Gander B. Collagen nerve conduits releasing the neurotrophic factors GDNF and NGF. Journal of Controlled Release: Official Journal of the Controlled Release Society. 2010;143(2):168-174.

26. Madduri S, Papaloizos M, Gander B. Synergistic effect of GDNF and NGF on axonal branching and elongation in vitro. Neuroscience Research. 2009;65(1):88-97.

27. Chen X, Wang XD, Chen G, Lin WW, Yao J, Gu XS. Study of in vivo differentiation of rat bone marrow stromal cells into schwann cell-like cells. Microsurgery. 2006;26(2):111-115.

28. Mills CD, Allchorne AJ, Griffin RS, Woolf CJ, Costigan M. GDNF selectively promotes regeneration of injury-primed sensory neurons in the lesioned spinal cord. Molecular and Cellular Neurosciences. 2007;36(2):185-194.

29. Boyd JG, Gordon T. Glial cell line-derived neurotrophic factor and brain-derived neurotrophic factor sustain the axonal regeneration of chronically axotomized motoneurons in vivo. Experimental Neurology. 2003;183(2):610-619.

30. Young S, Wong M, Tabata Y, Mikos AG. Gelatin as a delivery vehicle for the controlled release of bioactive molecules. Journal of Controlled Release: Official Journal of the Controlled Release Society. 2005;109(1-3):256-274.

31. Owens KW, Yukna RA. Collagen membrane resorption in dogs: a comparative study. Implant Dentistry. 2001;10(1):49-58.

32. $\mathrm{Ma} \mathrm{CH}$, Omura T, Cobos EJ, et al. Accelerating axonal growth promotes motor recovery after peripheral nerve injury in mice. The Journal of Clinical Investigation. 2011;121(11):4332-4347.
International Journal of Nanomedicine

\section{Publish your work in this journal}

The International Journal of Nanomedicine is an international, peerreviewed journal focusing on the application of nanotechnology in diagnostics, therapeutics, and drug delivery systems throughout the biomedical field. This journal is indexed on PubMed Central, MedLine, CAS, SciSearch ${ }^{\circledR}$, Current Contents ${ }^{\circledR} /$ Clinical Medicine,

\section{Dovepress}

Journal Citation Reports/Science Edition, EMBase, Scopus and the Elsevier Bibliographic databases. The manuscript management system is completely online and includes a very quick and fair peer-review system, which is all easy to use. Visit http://www.dovepress.com/ testimonials.php to read real quotes from published authors. 